\title{
É possível formar um cirurgião endovascular?
}

\author{
Eduardo Keller Saadi*
}

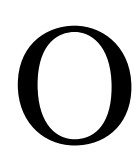
tratamento das doenças da aorta sempre foi alvo de ação da nossa especialidade.

Em 1991, Parodi realizou o primeiro implante de endoprótese no mundo para tratar um aneurisma de aorta abdominal [1].

Os recentes avanços em técnicas endovasculares como alternativa à cirurgia convencional para tratamento dos aneurismas e dissecções da aorta e seus ramos provocaram a criação de uma área específica denominada pela Associação Médica Brasileira de Cirurgia Endovascular.

A SBCCV se preocupa constantemente com a formação completa dos cirurgiões para todos os procedimentos relacionados à cirurgia cardiovascular, incorporando, na residência médica, o treinamento específico para procedimentos endovasculares, além do Departamento de Cirurgia Endovascular (DECEN) escrever diretrizes, proporcionar cursos de treinamento e educação continuada [2].

Quando novos procedimentos são introduzidos, a pressão por rápida implementação pode levar a desvios dos princípios fundamentais da formação médica, com risco de comprometimento da qualidade e segurança do atendimento ao paciente [3-5].

Para um profissional estar habilitado a realizar um procedimento endovascular da aorta, os critérios abaixo devem ser alcançados:

1) Conhecer profundamente a doença;

2) Tratar a doença com técnicas já estabelecidas;

3) Desenvolver habilidades técnicas;

4) Cuidar dos pacientes no pós-operatório;

5) Estar capacitado a tratar as complicações.

Como em qualquer procedimento, a qualificação técnica começa pelo embasamento teórico. Em primeiro lugar, é preciso conhecer a doença, a história natural, a fisiopatologia, os métodos diagnósticos de imagem, assim como os critérios de indicação cirúrgica [6,7]. Não se admite, hoje, um cirurgião que simplesmente execute tarefas discutidas e planejadas por outros.

É importante a experiência e o conhecimento do médico operador com outras técnicas, já bem estabelecidas para o tratamento das doenças da aorta, pois isto o ajudará na escolha do melhor método terapêutico adequado, reduzindo o viés de indicação. Cada vez mais, os procedimentos híbridos vêm ganhando espaço e somente os cirurgiões que dominarem as técnicas abertas, de circulação extracorpórea, além das endovasculares, serão capazes realizá-los com segurança.

A questão do desenvolvimento de habilidades técnicas para a realização do procedimento é apenas uma parte do problema. É evidente que, afora a aquisição de todo este armamentário teórico, o cirurgião deve desenvolver sua técnica de maneira absolutamente rigorosa e obstinada. $\mathrm{O}$ treinamento com exposição de vasos inguinais e retro-peritoniais, assim como o manejo de cateteres e guias, devem fazer parte do programa da residência médica.

Uma relação estreita com o paciente no pósoperatório é tão importante quanto antes e durante o procedimento. O cirurgião atual deve ser capaz de sustentar opiniões nas diversas fases da recuperação, desde a imediata até a tardia, no qual o acompanhamento periódico e por toda a vida é fundamental para evitar e tratar complicações, prevenindo a morte. Não há chance de aprendizado sem o acompanhamento sistemático dos pacientes para que se possa aferir o resultado do método a médio e longo prazo.

Quem realiza o procedimento endovascular deve estar suficientemente preparado para tratar as potenciais complicações. Os pacientes em que ocorra ruptura ou dissecção arteriais devem ser operados em regime de urgência absoluta, por profissionais que dominem ambas as técnicas. Embolizações periféricas requerem embolectomia, assim como oclusão de ramos arteriais necessita de cirurgia de revascularização imediata.

Na verdade, antes de ser cirurgião endovascular, é necessário ser cirurgião e, antes de tudo, é preciso ser MÉDICO COM VOCAÇÃO.

Com toda esta bagagem disponível, o assim chamado Cirurgião Endovascular só logrará êxito se conhecer profundamente a doença e estiver diretamente envolvido com o paciente. 
Muitos, mesmo sem conhecerem as complicações, podem ser treinados tecnicamente. Porém, sem o envolvimento com o doente, poderão, no máximo, serem bons operadores com habilidade manual e boa coordenação motora no manejo com cateteres, capazes de executar adequadamente as tarefas que, a rigor, poderiam ser aprendidas em uma escola de ofícios.

Os cirurgiões jovens devem iniciar o treinamento sob supervisão segura e atuante de especialistas competentes, para evitar que os pacientes corram riscos desnecessários.

Quem tem a responsabilidade de treinar pessoas deve sempre ter a preocupação com a qualidade do produto final do seu esforço, preservando a segurança dos pacientes.

A excelência no tratamento endovascular não é atingida somente com o treinamento inicial e a experiência clínica, mas com programas de educação continuada e, neste particular, a SBCCV vem desempenhando um papel de fundamental importância, que deve ser expandido e aperfeiçoado, principalmente em função da forte atuação de vários grupos de cirurgiões cardiovasculares nesta área e das enormes contribuições científicas realizadas tanto a nível nacional como internacional [2,8-12].

\section{* Presidente da Comissão de Defesa Profissional da SBCCV}

\section{REFERÊNCIAS}

1. Parodi JC, Palmaz JC, Barone HD. Transfemoral intraluminal graft implantation for abdominal aortic aneurysms. Ann Vasc Surg. 1991;5(6):491-9.

2. Albuquerque LC, Braile DM, Palma JH, Gomes WJ and Coselli J. Diretrizes para o tratamento cirúrgico das doenças da aorta da Sociedade Brasileira de Cirurgia Cardiovascular.”Guidelines for surgery of aortic diseases from Brazilian Society of Cardiovascular Surgery”. RBCCV 2006;21(1):1-23.

3. Kouchoukos N, Bavaria J, Coselli J, De La Torre R,
Ikonomidis J, Karmy-Jones R, Mitchell R, Shemin R, Spielvogel D, Svensson L and WheatleyG. Guidelines for credentialing of practitioners to perform endovascular stent-grafting of the thoracic aorta. Ann Thorac Surg 2006;81(3):1174-6.

4. Garrett HE Jr. Getting started with thoracic endografting. A cardiac surgeon's perspective on starting your practice. Endovasc Today 2005;4(Suppl):13-4.

5. Zipfel B, Hammerschmidt R, Krabatsch T, Buz S, Weng $\mathrm{Y}$ and Hetzer R. Stent-grafting of the thoracic aorta by the cardiothoracic surgeon. Ann Thorac Surg 2007;83(2):441-9.

6. Wolfe WG, Moran JF. The evolution of medical and surgical management of acute aortic dissection. Circulation. 1997;56(4pt 1):503-5.

7. Borst HG, Frank F, Schaps D. Treatment of extensive aortic aneurysms by a new multiple-stage approach. J Thorac Cardiovasc Surg. 1988;95(1):11-3.

8. Palma JH, Almeida DR, Carvalho AC, Andrade JC, Buffolo E. Surgical treatment of acute type B aortic dissection using an endoprosthesis (elephant trunk). Ann Thorac Surg. 1997;63(4):1081-4.

9. Buffolo E, Fonseca JHP, Souza JAM, Alves CMR. Revolutionary treatment of anuerysms and dissections of descending aorta: the endovascular approach. Ann Thorac Surg. 2002;74(5):S1815-7.

10.Palma JH, Souza JAM, Alves CMR, Carvalho AC. Selfexpandable aortic stent-grafts for treatment of descending aortic dissections. Ann Thorac Surg. 2002;73(4):1138-42.

11. Gabriel EA, Locali RF, Romano CC, Duarte AJ, Palma $\mathrm{JH}$, Buffolo E. Analysis of the inflammatory response in endovascular treatment of aortic aneurysms. Eur J Cardiothorac Surg 2007;31(3):406-12.

12. Palma JH, Geisthovel N, Brasil LA*, Ferrari Jr A., Carvalho AC,Gomes WJ, Buffolo E. Tratamento de aneurismas da parte torácica da aorta pela introdução de "stents" sob visão endoscópica.RBCCV 1998 13(1): 8-12. 